\title{
Recurrent carpal tunnel syndrome associated with extension of flexor digitorum muscle bellies into the carpal tunnel: A case series
}

\author{
Rochelle Castillo ${ }^{1}$, Khushboo Sheth ${ }^{2}$, Alan Babigian ${ }^{3}$, Christopher Scola ${ }^{4}$ \\ ${ }^{1}$ Department of Medicine, University of Connecticut, Farmington, $C T$; ${ }^{2}$ Division of Rheumatology and Immunology, Department of Medicine, \\ Stanford University, Stanford, CA; ${ }^{3}$ Section of Plastic/Reconstructive Surgery, Department of Surgery, Hartford Hospital, Hartford, CT; \\ ${ }^{4}$ Division of Rheumatology, Department of Medicine, Hartford Hospital, Hartford, CT, USA
}

While the success or failure of carpal tunnel release ultimately depends on the interplay of a wide array of factors, a broad understanding of the normal anatomy of the carpal tunnel accompanied by awareness of the possible variations of the individual structures that make up its contents is crucial to optimizing surgical outcomes. While anatomic variants such as extension of the flexor digitorum muscle bellies have been described as a cause of primary carpal tunnel syndrome (CTS), there have been no reports depicting its association with recurrent CTS following initially successful carpal tunnel release, a finding with potentially significant prognostic implications that can aid in operative planning. In such cases where muscle extension is identified preoperatively, careful debulking of the muscle belly may be beneficial in improving long-term surgical outcomes.

Keywords Carpal tunnel syndrome / Median nerve / Forearm / Hand / Reoperation
Correspondence: Christopher Scola Division of Rheumatology, Department of Medicine, Hartford Hospital, 80 Seymour St., Hartford, CT 06106, USA

Tel: $+1-860-246-4260$

Fax: +1-860-246-6020

E-mail: Christopher.Scola@

hhchealth.org

Received: 13 Apr 2017 • Revised: 20 Apr 2018 • Accepted: 25 Apr 2018

pISSN: 2234-6163 • elSSN: 2234-6171 • https://doi.org/10.5999/aps.2017.00724 • Arch Plast Surg 2018;45:474-478

\section{INTRODUCTION}

Carpal tunnel syndrome (CTS) is the most commonly diagnosed compression neuropathy of the upper extremity, affecting an estimated $4 \%$ to $7 \%$ of the general population [1]. Carpal tunnel release (CTR) has thus emerged as one of the most common hand operations performed worldwide, with the majority of cases resulting in significant improvement in symptoms. However, unsatisfactory postoperative outcomes such as persistence or recurrence can occur in up to $20 \%$ of cases, with up to $12 \%$ requiring reoperation $[2,3]$. While the success or failure of CTR ultimately depends on the interplay of a wide array of patient- or surgeon-derived factors, a broad understanding of the normal anatomy of the carpal tunnel accompanied by awareness of the possible variations of the individual structures that make up its contents is undoubtedly crucial to preventing postoperative complications and optimizing overall surgical outcomes. Such anatomic anomalies involving the flexor digitorum superficialis in particular have been noted as early as 1959 [4], with several case reports describing the association between extension of the flexor digitorum muscles into the carpal tunnel and development of entrapment mononeuropathy [5-7]. To date, however, there has been only one other account [8] of the association of flexor digitorum muscle extension into the carpal tunnel with 
recurrent CTS following initially successful CTR, a finding with potentially significant prognostic implications that can subsequently guide the approach to surgical management. The mechanism for how extension of a flexor digitorum muscle belly can cause residual or recurrent CTS postoperatively is illustrated in Fig. 1. In normal patients, the carpal tunnel should contain only tendons of the flexor muscles along with the median nerve (Fig. 1A). However, in patients with aberrant distal extension of a flexor digitorum muscle belly into the carpal tunnel, the tunnel becomes more crowded and can lead to residual or recurrent CTS after standard release (Fig. 1B). Herein, we present three cases of recurrent CTS following surgical release associated with

Fig. 1. A carpal tunnel following standard surgical release

Illustration of a carpal tunnel following standard surgical release in normal patients (A) and in patients with aberrant distal extension of a flexor digitorum muscle belly into the carpal tunnel (B), which can result in crowding of the carpal tunnel and recurrence of symptoms of carpal tunnel syndrome.
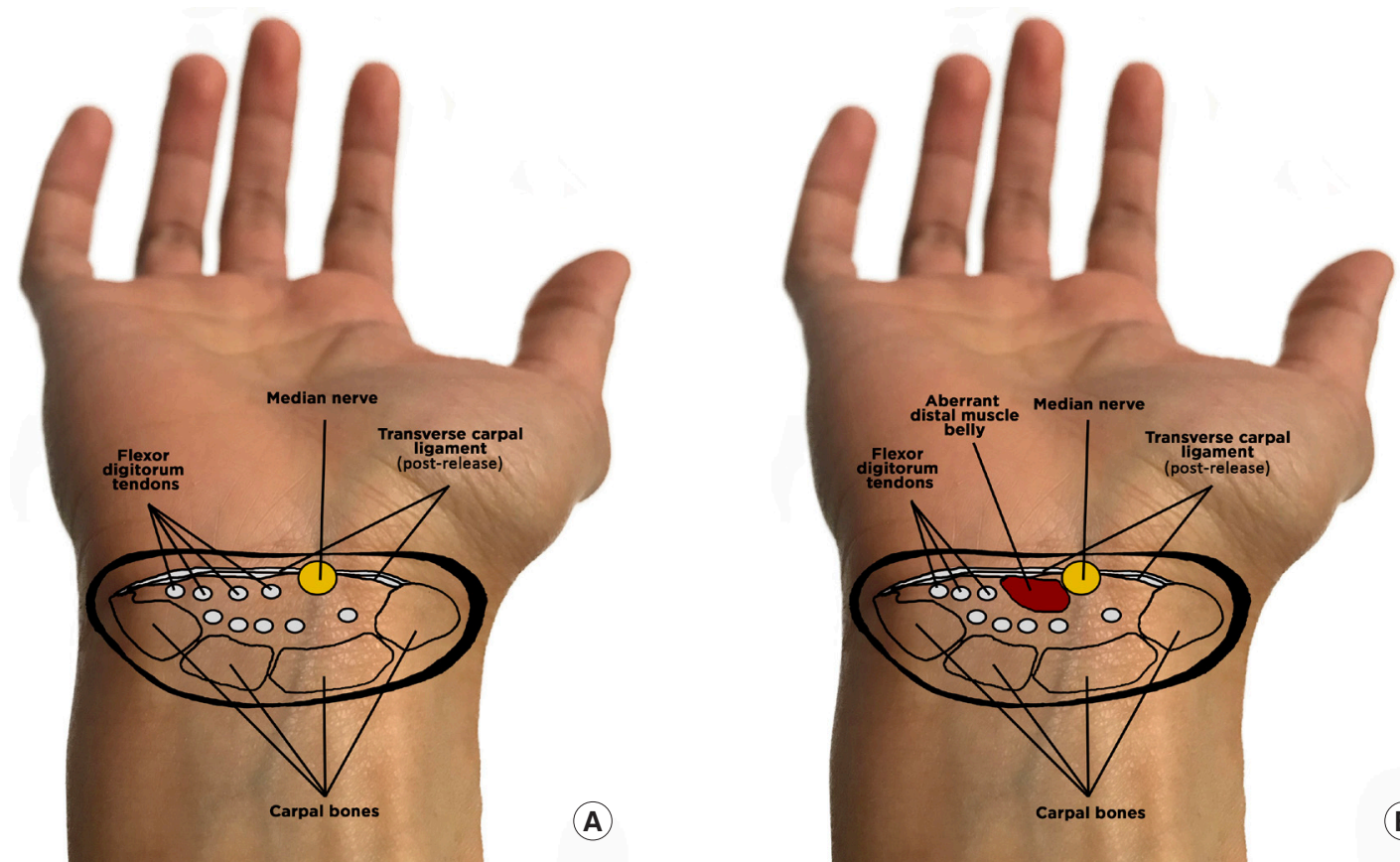

\section{Fig. 2. Musculoskeletal ultrasound: case 1}

Musculoskeletal ultrasound of the carpal tunnel at the wrist of a Hispanic female 1 month after carpal tunnel release. The second flexor muscle belly (arrow) can be clearly seen protruding through the carpal tunnel in proximity to the median nerve (asterisk). (A) Longitudinal view, (B) transverse view.
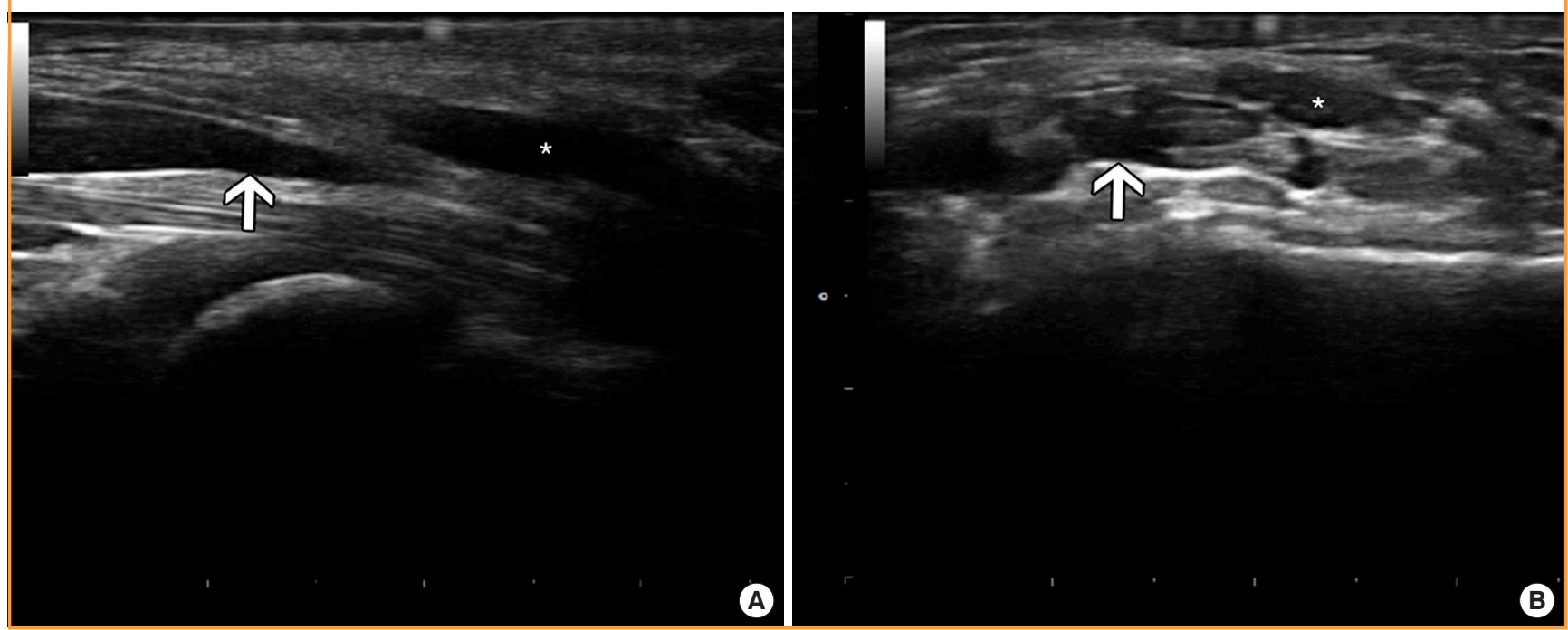
extension of the flexor digitorum muscle bellies into the carpal tunnel noted on musculoskeletal ultrasonography (MSK US).

\section{CASES}

\section{Case 1}

A 59-year-old Hispanic female with hypertension (HTN) and fibromyalgia presented with recurrent hand paresthesias 1 month after CTR. On physical examination, there was no synovitis of the wrists and work up for inflammatory arthritis was negative. Electromyogram and nerve conductions studies
(EMG/NCS) revealed bilateral moderate to severe CTS. MSK ultrasound exhibited extension of the second flexor digitorum muscle within the carpal tunnel (Fig. 2).

\section{Case 2}

A 68-year-old Caucasian female with a past medical history of HTN, coronary artery disease, and undifferentiated connective tissue disease characterized by an anti-nuclear antibody of 1:160, Raynaud's phenomenon, and arthralgias presented with pain and numbness on the right forearm and hand following CTR done 6 years ago. An EMG/NCS of the right hand con-

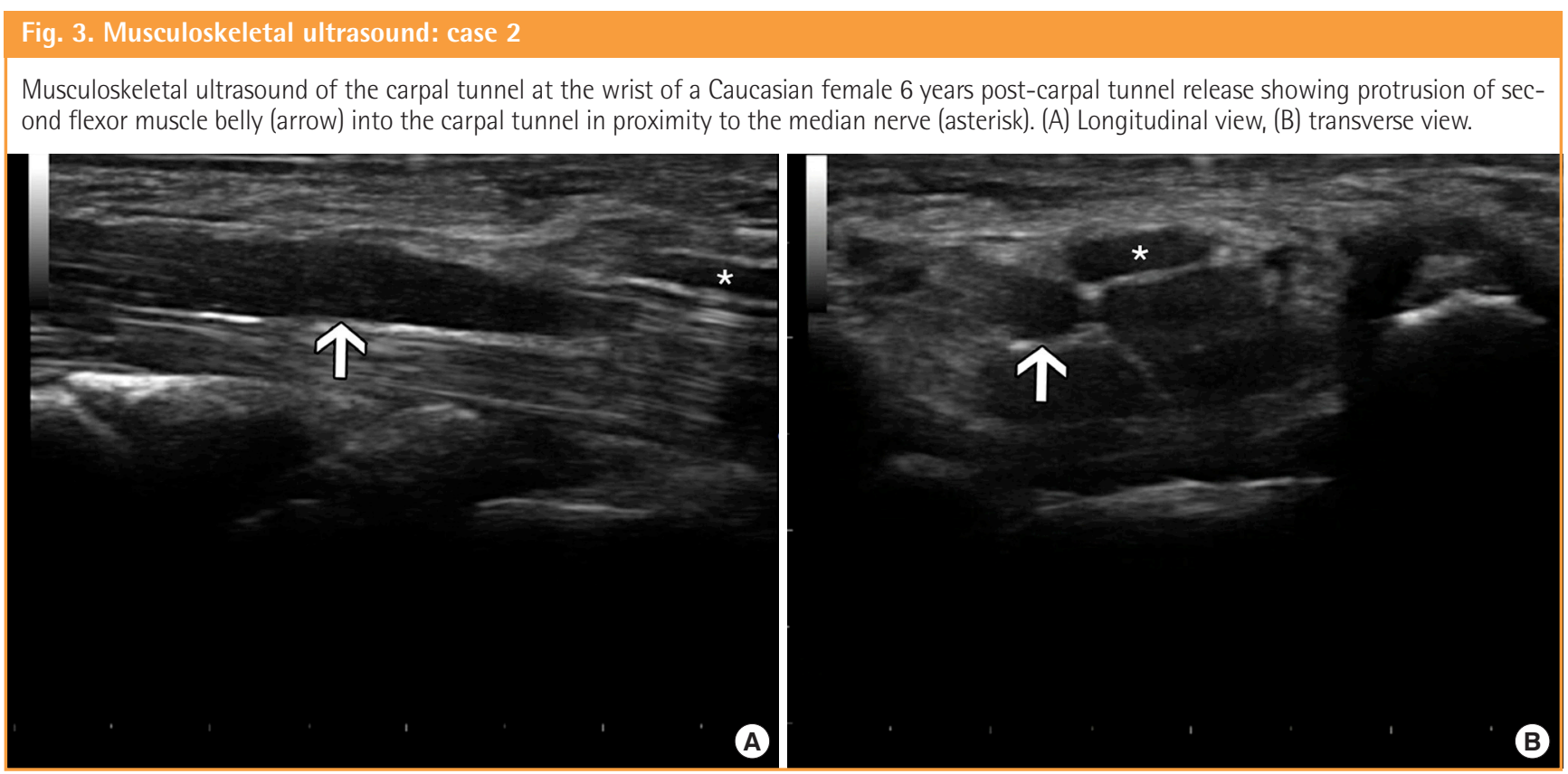

Fig. 4. Musculoskeletal ultrasound: case 3

Musculoskeletal ultrasound of the carpal tunnel at the wrist of an African-American female 20 years after carpal tunnel release depicting extension of the second flexor muscle belly (arrow) into the tunnel in proximity to the median nerve (asterisk). (A) Longitudinal view, (B) transverse view.
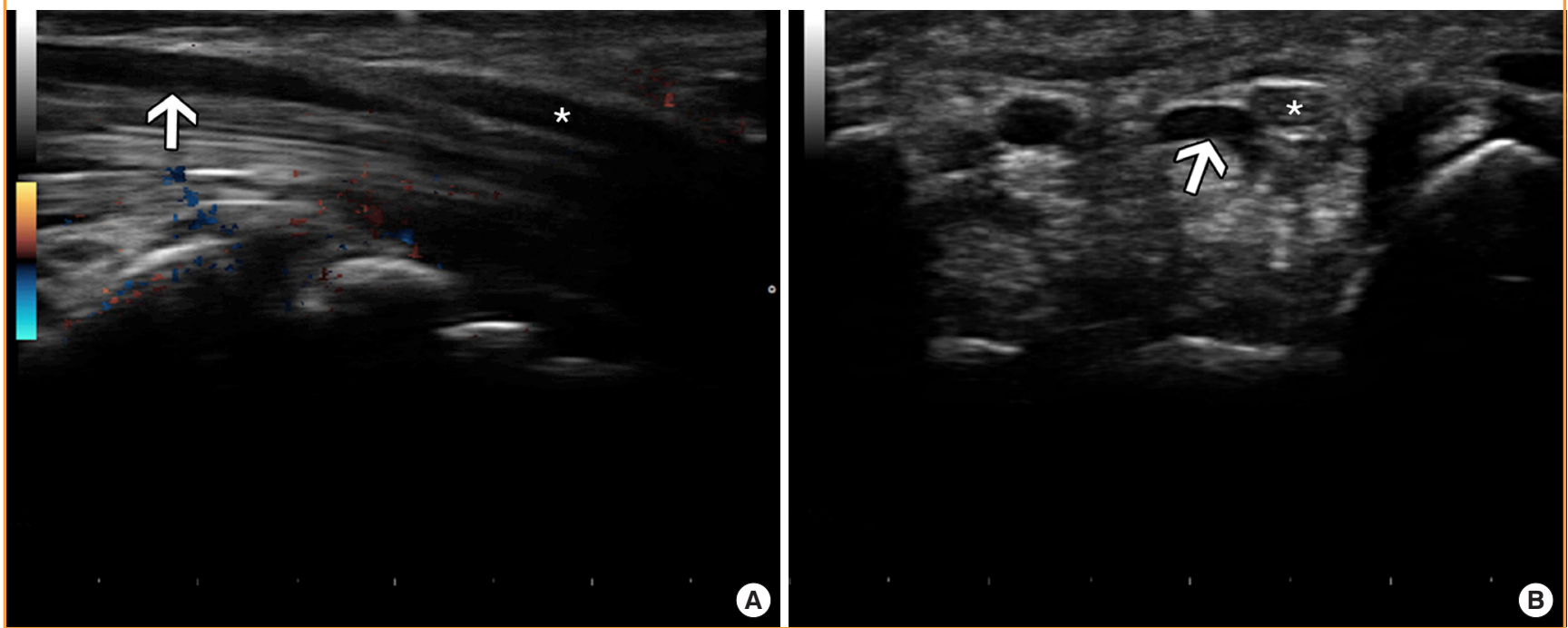
firmed recurrence of CTS. MSK US likewise showed presence of the second flexor digitorum muscle within the carpal tunnel (Fig. 3).

\section{Case 3}

A 53-year-old African-American female with seropositive rheumatoid arthritis well-controlled on disease-modifying antirheumatic drugs presented with recurrent bilateral CTS confirmed by EMG/NCS 20 years after undergoing bilateral CTR. MSK US depicted extension of flexor digitorum muscle bellies distally into the carpal tunnel (Fig. 4).

\section{DISCUSSION}

Recurrence of CTS following surgical release, defined as return of symptoms after a symptom-free interval of months to years following the index procedure, has been reported in $3 \%$ to $25 \%$ of cases [3]. The outcomes of revision surgery in the $12 \%$ or so that require surgical re-exploration have been generally disappointing with up to $40 \%$ reported as unfavorable and up to $95 \%$ of cases associated with a variety of persistent symptoms, making it particularly important to preoperatively ascertain potential modifiable factors that can lead to suboptimal outcomes [9].

The most frequently cited causes for recurrent CTS based on published retrospective studies looking at intraoperative findings during revision surgery include incomplete release of the flexor retinaculum, perineural fibrosis, scarring, and adhesions to the nerve. In terms of preoperative factors, a retrospective study done by Djerbi et al. [10] showed significantly higher rates of recurrence in patients with severe preoperative sensory deficit, known median nerve neuromas, workers compensation claims, and number of previous surgeries. A systematic review likewise showed poorer outcomes with diabetes mellitus, HTN, poor overall health status, thoracic outlet syndrome, double crush nerve injury, alcohol misuse, smoking, and noticeable preoperative wasting of the abductor pollicis brevis muscle [11].

Another often-overlooked area that has the potential to considerably affect the success of CTR is anatomical variations in the different components of the carpal tunnel. Variations in the median nerve and its branches are perhaps the most well-documented but other studies have cited deviations in bony (e.g., hook of hamate) and musculotendinous (e.g., lumbricals and palmaris longus) structures as predisposing factors. Anomalies of the flexor digitorum compartment in the hand have also been reported in the literature and range from the presence of anomalous accessory muscle bellies, to aberrant attachments between musculotendinous units, to extension of flexor digitorum muscle bellies into the proximal part of the carpal tunnel, as in our case series. The clinical consequences of these flexor digitorum muscle anatomic abnormalities are likewise broad and include the development of "trigger wrist," the appearance of painless or painful palmar masses, or the occurrence of CTS. The mechanism for the latter involves a simple increase in the volume of the carpal tunnel contents causing structural compression of the median nerve and development of CTS. A descriptive study performed on 28 female and 26 male cadavers showed a $46 \%$ prevalence of flexor digitorum superficialis and profundus muscle bellies in the carpal tunnel in female samples versus only $7.8 \%$ for the males [12]; whether this can be directly linked to the increased prevalence of CTS in females compared to males remains to be fully elucidated. A few other published cases have reported on CTS caused by extension of normal flexor digitorum muscle bellies distally into the carpal tunnel in the absence of a distinct palmar mass. As early as 1959, Tanzer [4] reported on intraoperative findings of the muscle belly of the flexor digitorum superficialis to the index finger extending through the carpal tunnel in two patients. More recently, Kerasnoudis [5] described an elongated muscle belly of the flexor digitorum superficialis in the carpal tunnel of a 46-year-old farmer causing CTS that was treated conservatively. The only other account of recurrent CTS following CTR attributed to an anomalous flexor digitorum superficialis (FDS) muscle belly extending through the carpal tunnel was reported by Schon et al. [8]. In this patient, resolution of symptoms was noted postoperatively with excision of the muscle bellies, which further supports a potential association between extension of the FDS muscle into the carpal tunnel with recurrent CTS following initially successful decompression surgery. Thus, in patients with protrusion of normal flexor digitorum muscle bellies into the proximal carpal tunnel, it may be prudent to perform careful debulking of any muscle belly that may be present within the carpal tunnel whilst maintaining integrity of the tendon itself, which is best accomplished through an open approach. Further research on such an approach would be of interest in this clinical setting.

MSK US is an easy, inexpensive, noninvasive method of imaging the carpal tunnel and helping to preoperatively determine anatomical deviations that can impact surgical outcomes. It is significantly more affordable than other imaging modalities such as magnetic resonance imaging and can be done in-office. Dynamic testing can identify such anatomic abnormalities by having patients flex and extend the digits while imaging the carpal tunnel with ultrasound. In our case series, all the patients demonstrated protrusion into the carpal tunnel of the index flexor muscle bellies upon full extension of the index finger and recession of the muscle belly out of the carpal tunnel space with full digit flexion. Thus, the usefulness of routinely incorporating 
MSK US in diagnosing CTS and assessing carpal tunnel anatomy to aid in both steroid injections and surgical planning cannot be overemphasized. Such dynamic testing may identify patients who are at risk for suboptimal surgical outcomes with routine release procedures. If the relatively low cost of US remains prohibitive, an option would also be to perform ultrasound only on patients with recurrent CTS to detect if this anatomic variation is present and may be responsible for recurrence of symptoms. Performing US on a narrower patient population will thus increase the possible cost effectiveness of this test. If extension of FDS muscle into the carpal tunnel is encountered intraoperatively, a potential surgical solution would be careful debulking in addition to transecting the transverse carpal ligament.

In summary, we presented a case series of three females presenting with ultrasound-proven distal extension of the flexor digitorum muscle bellies into the carpal tunnel and clinical and electromyographic evidence of recurrent CTS after an initial symptom-free period. The importance of this case series lies not only in its uniqueness but also in suggesting the role of distal extension of normal flexor digitorum muscle bellies into the carpal tunnel as a potential causative factor for recurrent CTS and the usefulness of MSK US in both diagnosing this unusual condition and subsequently aiding in the selection of the most optimal therapeutic approach. We acknowledge that one shortcoming of the study is that in each of the cases, the CTR procedure preceded the identification of the anatomic abnormality visualized on ultrasound imaging and therefore intraoperative photographs were unavailable. We would not expect, however, that the additional information of intraoperative photographs would provide discordant information given the reliability of ultrasound imaging. Future studies can focus on identifying such anatomic variations preoperatively and comparing the outcomes of those undergoing routine CTR with those in whom muscle debulking is also performed.

\section{NOTES}

\section{Conflict of interest}

No potential conflict of interest relevant to this article was reported.

\section{Patient consent}

The patients provided consent for the performance of ultrasonography and had no objections to the use of the ultrasound images for purely academic purposes. All identifying information was omitted and complete anonymity of the subjects was maintained in the writing of this manuscript.

\section{REFERENCES}

1. Kleopa KA. Carpal tunnel syndrome. Ann Intern Med 2015; 163:ITC1.

2. Beck JD, Brothers JG, Maloney PJ, et al. Predicting the outcome of revision carpal tunnel release. J Hand Surg Am 2012; 37:282-7.

3. Jones NF, Ahn HC, Eo S. Revision surgery for persistent and recurrent carpal tunnel syndrome and for failed carpal tunnel release. Plast Reconstr Surg 2012;129:683-92.

4. Tanzer RC. The carpal-tunnel syndrome; a clinical and anatomical study. J Bone Joint Surg Am 1959;41-A:626-34.

5. Kerasnoudis A. Elongated muscle belly of the flexor digitorum superficial causing carpal tunnel syndrome. Hand (N Y) 2012;7:333-4.

6. Smith RJ. Anomalous muscle belly of the flexor digitorum superficialis causing carpal-tunnel syndrome: report of a case. J Bone Joint Surg Am 1971;53:1215-6.

7. Hutton P, Kernohan J, Birch R. An anomalous flexor digitorum superficialis indicis muscle presenting as carpal tunnel syndrome. Hand 1981;13:85-6.

8. Schon R, Kraus E, Boller O, et al. Anomalous muscle belly of the flexor digitorum superficialis associated with carpal tunnel syndrome: case report. Neurosurgery 1992;31:96970.

9. Tollestrup T, Berg C, Netscher D. Management of distal traumatic median nerve painful neuromas and of recurrent carpal tunnel syndrome: hypothenar fat pad flap. J Hand Surg Am 2010;35:1010-4.

10. Djerbi I, Cesar M, Lenoir H, et al. Revision surgery for recurrent and persistent carpal tunnel syndrome: clinical results and factors affecting outcomes. Chir Main 2015;34: 312-7.

11. Turner A, Kimble F, Gulyas K, et al. Can the outcome of open carpal tunnel release be predicted? A review of the literature. ANZ J Surg 2010;80:50-4.

12. Holtzhausen LM, Constant D, de Jager W. The prevalence of flexor digitorum superficialis and profundus muscle bellies beyond the proximal limit of the carpal tunnel: a cadaveric study. J Hand Surg Am 1998;23:32-7. 